\title{
Extreme solar storms based on solar magnetic field
}

\author{
Brigitte Schmieder ${ }^{1}$ \\ Observatoire de Paris, LESIA, 5 place Janssen, 92195 Meudon, France. \\ ${ }^{a}$ PSL Research University, CNRS, Sorbonne Universités, UPMC Univ. Paris 06 \\ ${ }^{b}$ Univ. Paris Diderot, Sorbonne Paris Cité
}

\begin{abstract}
Many questions have to be answered before understanding the relationship between the emerging magnetic flux through the solar surface and the extreme geoeffective events. Which threshold determines the onset of the eruption? What is the upper limit in energy for a flare? Is the size of sunspot the only criteria to get extreme solar events?

Based on observations of previous solar cycles, and theory, the main ingredients for getting X ray class flares and large Interplanetary Corona Mass Ejections e.g. the built up of the electric current in the corona, are presented such as the existence of magnetic free energy, magnetic helicity, twist and stress in active regions. The upper limit of solar flare energy in space research era and the possible chances to get super-flares and extreme solar events can be predicted using MHD simulation of coronal mass ejections.
\end{abstract}

Keywords: Solar flares, Stellar flares

\section{Introduction}

Extreme solar storms can be defined as energetic solar events related to large-scale disturbances in the Earth's magnetosphere, called as geomagnetic events (Cliver and Svalgaard, 2004, Koskinen and Huttunen, 2006: Echer et al. 2011b a, 2013, Gonzalez et al., 2011b). Before the launch of satellites, the

\footnotetext{
* Corresponding author

Email address: brigitte.schmieder@obspm.fr (Brigitte Schmieder)
} 
activity of the Sun was recorded by ground-based instruments observing in visible light (e.g. see the Meudon data-base "BASS2000" with spectroheliograms registered from 1909 until today- see examples in Figure 1). Surveys in white light, in $\mathrm{H} \alpha$, and $\mathrm{Ca}$ II $\mathrm{H}$ and $\mathrm{K}$ lines allow to study the solar cycle activity by tracking the sunspots and studying their size, and their complexity (Waldmeier, 1955, McIntosh, 1990; Eren et al., 2017). The enhancement of emission was used as a good proxy for detecting flares (Carrington, 1859). However the detection of flares was limited by the spatial and the temporal resolution of the observations.

Recently different approaches have succeeded to quantify the intensity of some historical events using different magnetometer stations over the world. The analysis of magnetic recordings made as early as the middle of the nineteenth century by ground stations allowed us to clarify the importance of several extreme events (Tsurutani et al., 2003, Cliver and Svalgaard, 2004, Lakhina et al. 2008, Cid et al., 2013, Cliver and Dietrich, 2013). During the $\mathrm{XX}^{\text {th }}$ century, several important events with $D s t<-700 \mathrm{nT}$ were observed after intense flares and connected to aurora. Exploring historical extreme events shows all the problems encountered when one aims at understanding the phenomena from one end to the other. It is difficult to identify the solar source of extreme geoeffective events without continuous observations of the Sun and without quantified numbers of the energy release during the solar events.

The Geostationary Operational Environmental Satellites (GOES) register the global soft X ray emission $1-8 \AA$ of the Sun since the " 80 s". The intensity of the flares are classified by the letters X, M, C, which correspond to $10^{-4}, 10^{-5}$, $10^{-6} \mathrm{~W} \mathrm{~m}^{-2}$ energy release respectively. The extreme historical solar events, for which only the size of sunspots and "the magnetic crochet" recorded on the Greenwich magnetogram, for example, for the Carrington event or ionospheric disturbances are known, were associated with extreme geomagnetic events by comparison with recent events. It is interesting to read the papers of Tsurutani et al. (2003); Cliver and Dietrich (2013) where several historical events e.g. Sept. 1859, Oct. 1847, Sept.1849, May 1921 have been discussed and classified. 
With the SOlar and Heliospheric Observatory (SOHO; Fleck et al., 1995), launched in 1995, and its on-board spectro-imagers and coronagraphs, and more recently with the Solar TErrestrial RElations Observatory (STEREO A and B 2006; Wuelser et al., 2004; Russell, 2008 and its COR and HI coronagraphs able to reach the Earth in particular conjunction (see the website of HI HELCATS) the solar sources of geoeffective events could be identified with more accuracy. A new era was open for forcasting geomagnetic disturbances by being able to follow the solar events in multi-wavelengths, and particularly the coronal mass ejections from the Sun to the Earth. This is the new science called "Space Weather". Intense flares responsible for geoeffective events are commonly associated with Solar Energetic Particles (SEP) events and/or coronal mass ejections (CMEs). Several minutes after the flares, very high energetic particles (SEPs) may enter in the Earth's atmosphere affecting astronauts or electronics parts in satellites. However, concerning geomagnetic disturbances, CMEs can be as geoeffective as the energetic particles when their arrival trajectory is oriented towards the Earth and when their speed is large enough (Gopalswamy et al. 2010a b; Wimmer-Schweingruber, 2014). SEP ejections produce particle radiation with large fluence, however only a few of SEPs occur during each solar cycle while CMEs have an occurrence rate between 2 and 3 per week in solar minimum and between 5 and 6 per day in solar maximum, these numbers also depend on the used coronagraphs (St. Cyr et al. 2000, Webb and Howard, 2012 , Lugaz et al. 2017). They are originated from highly-sheared magnetic field regions which can be refereed as large magnetic flux ropes carrying strong electric currents. They are statistically more likely to lead to geomagnetic disturbances when their solar sources are facing the Earth (Bothmer and Zhukov, 2007; Bein et al., 2011; Wimmer-Schweingruber, 2014). According to their speed, their interplanetary signatures (ICMEs) may reach the Earth in one to five days after the flare (Yashiro et al. 2006: Gopalswamy et al., 2009, Bein et al., 2011).

Halo CMEs observed with the white light SMM coronagraph were firstly named "global CMEs" Dere et al. (2000) and already suspected to be responsible of geoeffective events (Zhang and Burlaga, 1988). Recent studies confirmed the 
geoeffectivity of halo CMEs which generally form magnetic clouds (MC) (e.g. Bocchialini et al 2017, Solar Physics in press). The MCs are associated with extreme storms $(D s t<-200 \mathrm{nT})$ and intense storms $(-200<D s t<-100 \mathrm{nT})$ (Gonzalez et al. 2007, Zhang et al., 2007), while the moderate storms $(-100<$ $D s t<-50 \mathrm{nT})$ studied in the solar cycle 23 were found to be associated with co-rotating regions by $47.9 \%$, to ICMEs or magnetic clouds (MC) by $20.6 \%$, to sheath fields by $10.8 \%$, or to combinations of sheath and ICME (10\%) (Echer et al. 2013).

However magnetic clouds can be not so effective if they are directed away from Earth like the fast ICME of July 2012 (Baker, 2013) or if the magnetic field of the cloud arrives close to the magnetosphere with an orientation towards the North as for the cases of August 1972 (Tsurutani et al. 1992). In August 1972 a huge sunspot group McMath region 11976 (see Figure 1) crossed the disk and was the site of energetic flares and consequently shocks were detected at 2.2 AU by Pionneer 10 (Smith, 1976). The estimated velocity of the ejecta was around $1700 \mathrm{~km} / \mathrm{s}$ which is nearly the highest transit speed on record. Tsurutani et al. (2003) estimated its magnetic field to be around $73 \mathrm{nT}$ which is also a huge number. But the Dst index indicated a recovery phase relatively low like a moderate storm (Tsurutani et al., 1992). Nowaday the in situ parameters of the solar wind including the interplanetary magnetic field, IMF, are monitored at L1 by the ACE spacecraft (Chiu et al., 1998) magnetic field (MAG experiment) or similar instruments. They indicate clearly the passage of the satellite through an ICME or magnetic cloud by the changes of the solar wind speed, the reversed sign of the magnetic components Bx and By. The ICME is more geoeffective if the IMF-Bz component is negative indicating a strong coupling with the magnetosphere.

We can conclude that if extreme solar storms do not necessary initiate extreme geomagnetic events, extreme geomagnetic events are nearly always produced by extreme solar storms. And extreme solar storms are most of the time issued from the biggest sunspot groups which produce the most energetic events (Sammis et al. 2000). 
The paper is organized as following. After an historical review of large sunspot groups observed on the Sun related to geomagnetic storms (Section 2), we present statistical results on star and sun flares according to the characteristics of the spots (flux, size) (Section3). Section 4 is focused on a MHD model (OHM) predicting the capability of the Sun to produce extreme events. Finally the conclusion is given in Section 5.

\section{Historical view of solar sources of geoeffectivity}

The Carrington event in September 1, 1859, well known to be one of the largest solar Sunspot groups leading to one of the strongest flare Carrington. 1859 , Hodgson, 1859) had the largest magnetic signature ever observed at European latitudes with the consequent aurora visible at low geographic latitude $\left( \pm 18^{\circ}\right)$ observed 17.5 hours later. Using the transit time, Tsurutani et al. (2003) proposed that the Dst value decreased down to $-1760 \mathrm{nT}$ during this event. The Colaba (Bombay) record allowed to have a more precise determination around $-1600 \mathrm{nT}$ (Cliver and Dietrich, 2013, Cid et al., 2013). This value is more than twice the value of the next extreme geomagnetic events. Revisiting this event by analysing ice core nitrates and ${ }^{10} \mathrm{Be}$ data, Cliver and Dietrich (2013) claimed that it reached only $-900 \mathrm{nT}$. Nevertheless it seems to be the strongest geoeffective event registered up to now. A correlation between solar energetic proton fluence (more than $30 \mathrm{MeV}$ ) and flare size based on modern data proves that this event can be classified as an extreme solar event with a X-ray flare having an estimated energy larger than X10. All these extreme registered events, 12 episodes since the Carrington events, are solar activity dependent (Gonzalez et al., 2011a) (rough association). They occurred mainly during solar cycle maximum of activity with its two bumps and a secondary peak during the declining phase of the solar cycle.

Between 1876 and 2007, the largest sunspot area overlaid by large bright flare ribbons was observed in the Meudon spectroheliograms in Ca II K1v and H $\alpha$ between July 20-26 1946 (Dodson and Hedeman, 1949). A well observed 

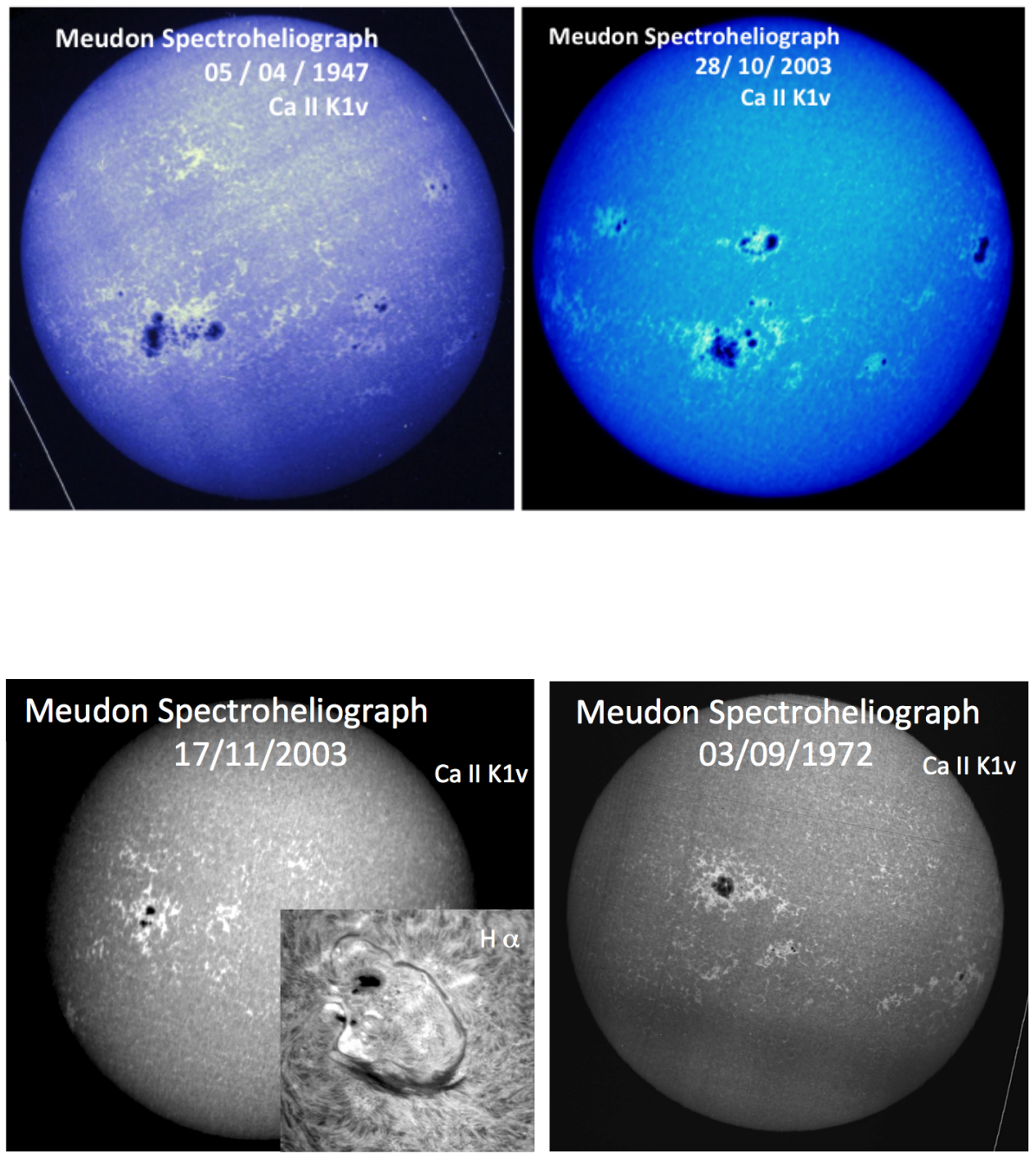

Figure 1: Full disk spectroheliograms from the Observatoire de Paris in Meudon. (top panels) The largest sunspot groups ever reported: (left) on April 4, 1947 with no geoffective effect, (right) on October 28 2003. The AR 10486 in the south hemisphere led to a X17 flare and consequently a geomagnetic disturbance with a Dst=-350 nT.(bottom panels): (left) AR 10501 on November 172003 observed in Ca II KIv with an inserted H $\alpha$ image of the active region. The huge eruptive filament surrounding the AR initiated the largest Dst of the $23^{\text {th }}$ solar cycle (Dst=-427 nT). (right) McMath region 11976 large sunspot, source of flares and ejected energetic particles on August 1972 (spectroheliograms from the Meudon data-base "BASS2000"). 


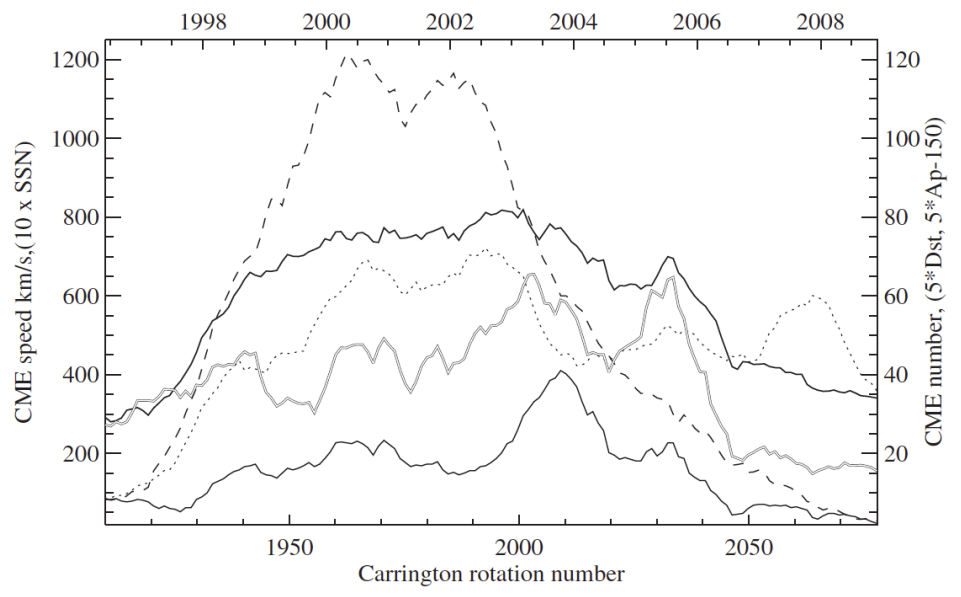

Figure 2: CME number and speed per solar Carrington rotation related to sunspot number and indexes of geoeffectivity (Dst and Ap). The dashed line shows the sunspot number, the bold solid line the CME speed index, the dotted line the CME number, the double line the Dst index, and the thin solid line represents the Ap index (adapted from Kilcik et al. (2011)).
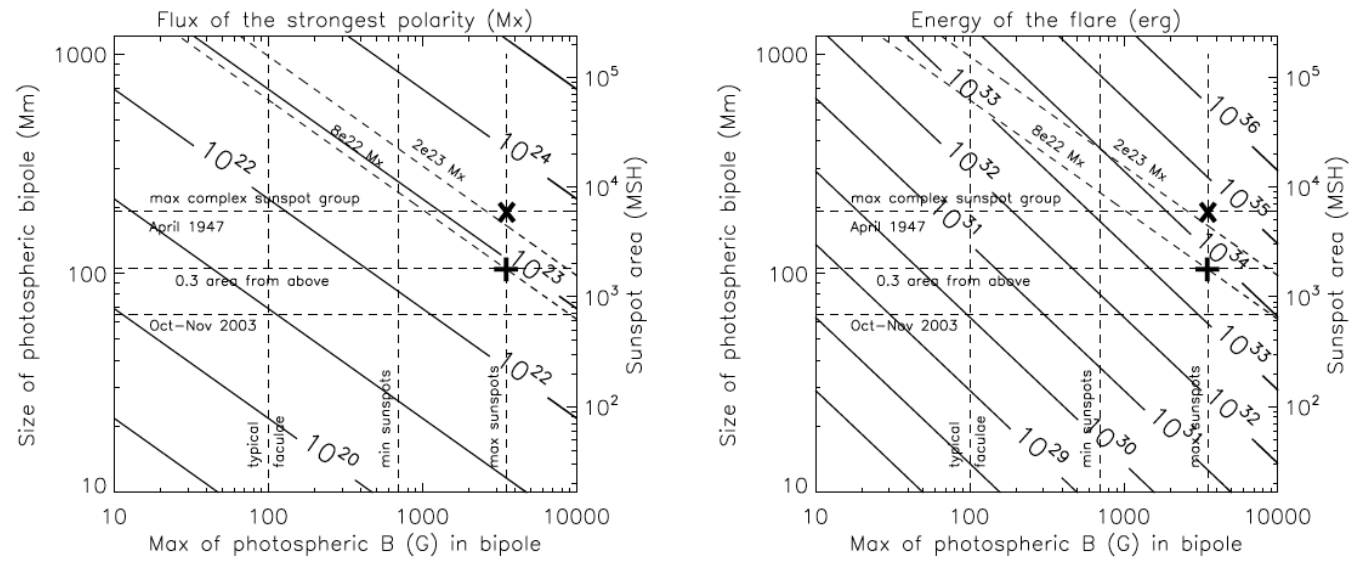

Figure 3: Magnetic flux in the dominant polarity of the bipole, and magnetic energy released during the flare, calculated as a function of the maximum magnetic field and the size of the photospheric bipole. The $\mathrm{x}$ and + signs correspond to extreme solar values. The former is unrealistic and the latter must be very rare (from Aulanier et al. (2013).) 
flare event occurred on July 251946 at 17:32 UT and caused a huge geomagnetic storm 26.5 hours later. The size of the sunspot was equivalent to 4200 millionths of the solar hemisphere (MSH) and the ribbon surface around 3570 MSH (Toriumi et al., 2016); The Carrington AR sunspot group seemed to be smaller than that one according to the sunspot drawings.

The next year an even larger sunspot was visible in the spectroheliogram of April 5, 1947 with a size reaching $6000 \mathrm{MSH}$ but had no geoeffectivity effect (Figure 1). The flare looked to be extended and powerful but not accompanied by coronal mass ejections. It could be a similar case to the more recently event observed in October 2014. The AR 12192 presented a sunspot area of 2800 MSH and was the site of several flares (6 X- and 24 M-class) (Sun et al., 2015. Thalmann et al. 2015). These two active regions are really exceptional. The AR 12192 did not launch any CMEs. Different interpretations have been proposed: the region would possess not enough stress, no enough free energy. Or the CME eruptive flux rope would not have reached the threshold height of the torus instability (Zuccarello et al., 2015).

Although there are in average two CMEs per day, only some of them are geoeffective. In October and November 2003, the largest sunspot groups (AR 10486 with an area of $3700 \mathrm{MSH})$, crossed the disk and were the sites of extreme events (Figure 1). X 17, X 10 and X 35 flares were reported on October 28, October 29 and November 4 respectively. However the more extreme geomagnetic storm occurring during the whole Solar Cycle 23 with a $D s t=-422$ nT was linked to a M9.6 class flare on November 20, 2003 Gopalswamy et al. 2005, Möstl et al., 2008, Marubashi et al., 2012). The origin of the solar event was in the region AR 10501 and has been associated with the eruption of a large filament (Chandra et al., 2010) (Figure 1). The AR 10501 had not the largest sunspot area but the cause of the flare and CME was merely due to the injection of opposite magnetic helicity by a new emerging flux which produced a destabilization of the large filament and lead to a full halo CME ( speed $=690$ $\mathrm{km} / \mathrm{s}$ ) and a magnetic cloud in the heliosphere. The size of the sunspot is an important parameter but it is not sufficient to get an extreme solar storm. 
Since the geoeffectivity is not straightforward, in order to forecast major storms, it is important to understand the nature (magnetic strength and helicity) and the location of the solar sources, the propagation of the CMEs through the interplanetary medium and their impacts on the magnetosphere/ionosphere system. Statistical studies of solar and magnetic activities during solar cycle 23 have permitted to associate CMEs and geomagnetic disturbances, providing long lists of CMEs with their characteristics i.e. their width, velocity, and solar sources (Zhang et al., 2007, Gopalswamy et al., 2010a b). They showed that a CME would more likely give rise to a geoeffective event if its characteristics are: a fast halo CME (with an apparent width around $360^{\circ}$ ) and a solar source close to the solar central meridian.

In some cases, the proposed sources came from active regions close to the limb. Cid et al. (2012) proposed to revisit this subset of events: in order to associate every link in the Sun-Earth chain, they have not only considered the time window of each CME-ICME, but also they have carefully revised every candidate at the solar surface. The result was that a CME coming from a solar source close to the limb cannot be really geoeffective (i.e, associated with a at least moderate and a fortiori intense storm) if it does not belong to a complex series of other events. Possible deflection of a CME in the corona as well as in the interplanetary space may change the geoeffectiveness of a CME Webb and Howard, 2012). It has been reported deflection up a few ten degrees, even during the SMM mission (Mein et al., 1982, Bosman et al., 2012; Kilpua et al., 2009. Zuccarello et al. 2012, Isavnin et al., 2013, Möstl et al., 2015). In the statistical analysis of Bocchialini et al 2017, it has been shown that a CME deflected from its radial direction by more than 20 degrees produced an exceptional geoeffective event. Moreover the orientation of the magnetic field of the magnetic cloud $(B z<0)$ is also an important parameter to get an extreme geoffective event (see the Introduction). 


\section{Characteristics of super flares}

Free magnetic energy stored in the atmosphere is released through global solar activity including CMEs (kinetic energy), flares and SEPs (thermal and non thermal energy). There is not really a physical reason to have a relationship between the different categories of released energy. Emslie et al. (2012) estimated all energy components for 38 solar eruptive flares observed between 2002 and 2006. The maximum of non potential energy in an active region reached $3 \times 10^{33} \mathrm{erg}$ and therefore could power all flare activity in the region. 0.5 percent of CMEs have a kinetic energy reaching $3 \times 10^{32} \mathrm{erg}$, otherwise the mean kinetic energy of 4133 CMEs is around $5 \times 10^{29} \mathrm{erg}$. They found a weak relationship between the estimations of the different energies due to large uncertainties. However the relationship looks to be more reliable for extreme events (syndrome of the big flare). However the systematic study of geoeffective events occurring through the solar maximum activity year (2002) already mentioned in Section 1 , showed that only $2 \mathrm{X}$-class flares among the $12 \mathrm{X}$-class flares were related to Sudden Storm Commencement (SSC) leaded events in the magnetosphere, the other SSCs were related to M and even C class flares (Bocchialini et al 2017). The solar cycle variation of the Dst does not follow the general trend of the sunspot number during the declining phases of solar cycles but is comparable to the trend of CME speeds, and CME numbers with the secondary peak (Kilcik et al. 2011) (Figure 22). This behaviour confirmed the importance of CME in the geoeffectivity.

However statistical analysis of flare intensity showed a relationship with some categories of active regions. Flares were related to large sunspot active regions (category A, B, F ) in the classification of Zurich (Eren et al. 2017). The class $\mathrm{F}$ consists of large ARs with sunspot fragmentation, indicating commonly the existence of strong shear. This study confirmed the finding concerning

the historical events that large geoffective effects are linked to the existence of large sunspot groups (Carrington, 1859, Dodson and Hedeman, 1949). The 
extreme events should be related to large sunspots like for the "Halloween" events on October-November 2003 in AR 10486 (Figure 1 top right). The flare on November 4 2003, is generally considered to be the most intense SXR event during the space age, with an estimated peak SXR classification ranging from X25 to X45 (Gopalswamy et al., 2005, Cliver and Dietrich, 2013). However the most geoeffective event occurred on the 20 November 2003. The AR 11501 has not a large sunspot and the solar extreme event is a coronal mass ejection with large kinetic energy. This event shows one example of large geoffectivity not related to the sunspot size (Figure 1 bottom row) but to the magnetic shear and magnetic helicity injection (Chandra et al. 2010).

Recently super flares (energy $10^{34}$ to $10^{36} \mathrm{erg}$ ) have been discovered in Sunlike stars (slow rotating stars) by the Kepler new space satellite (Maehara et al. 2012). A debate started about the possibility of observing such super flares on the Sun. Shibata et al. (2013) forecasted that one such super flare could occur every 800 years. Stars are suspected to have large spots and a large sunspot on the Sun with a flux of $2 \times 10^{23} \mathrm{Mx}$ flux would be not impossible and would correspond to an energy of $10^{34} \mathrm{erg}$ (Shibata et al. 2013).

Toriumi et al. (2016) made a statistical analysis of the new solar Cycle 24 flares between May 2010 and April 2016. Considering 51 flares exhibiting two flare ribbons (20 X and $31 \mathrm{M}$-class), they determined an empirical relationship between the size of sunspots $\left(\mathrm{S}_{\text {spot }}\right)$ in flaring active regions and the magnetic flux $\Phi_{\text {spot }}$ in logarithm scale.

$\log \Phi_{\text {spot }}=0.74 \times \log \mathrm{S}_{\text {spot }}+20$ with some uncertainties.

Considering the largest spots ever observed on the Sun (July 1946 and October 2014) they extrapolated this relationship and estimated a maximum flux of $1.5 \times 10^{23} \mathrm{Mx}$. They did not take into account the fact that all the energy of the spots can be transformed in thermal and non thermal energy and not in kinetic energy (no CME was launched in October 2014 for example). 


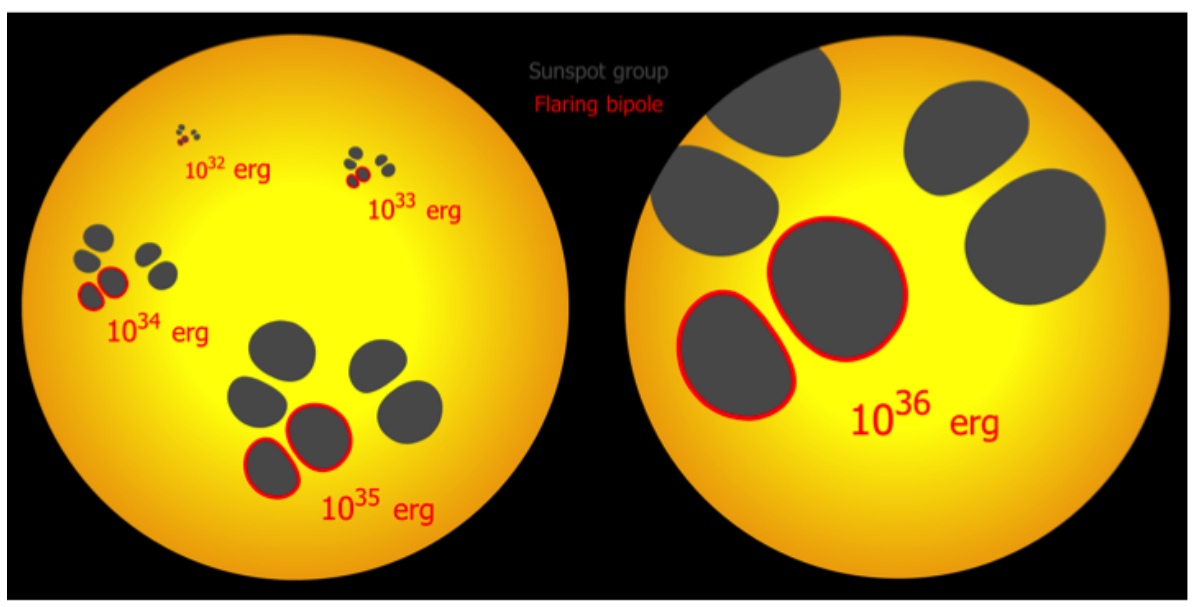

Figure 4: Schematic representation of several modeled sunspot groups without faculae on the solar disk, with their corresponding modeled flare energies computed with the OHM simulation. A sunspot group consists of several pairs of sunspots. In each group a pair of sunspots (surrounded by red curve) representing $1 / 3$ of the sunspot group area, is modeled in the simulation. The size of the grey areas is normalized to the size of the spots considered in the simulation (adapted from Aulanier et al. (2013)). 


\section{Prediction of extreme solar storms}

It appears that MHD simulations of emerging flux could be used to have a systematic survey to investigate the process of energy storage and find the relationship between sunspot size, CME eruptive events. The Observationally driven High order scheme Magnetohydrodynamic code (OHM) (Aulanier et al. 2005, 2010) simulation has been used as a tool to experiment huge energetic events on the Sun e.g. large super flare $\left(10^{36} \mathrm{erg}\right)$ by varying the characteristics of the sunspots in a large parameter space (Aulanier et al. 2013). The model consisted of a bipole with two rotating sunspots which is equivalent to create along the polarity inversion line a strong shear with cancelling flux. The 3D numerical simulation solved the full MHD equations for the mass density, the fluid velocity $\mathrm{u}$, and the magnetic field $\mathrm{B}$ under the plasma $\beta=0$ assumption. The calculations were performed in non-dimensionalized units, using $\mu=1$. The magnetic field diffusion favored the expulsion of the flux rope. The space parameter study lead to graphs of values of magnetic flux and energy according to the size of sunspot in MSH units and the stress of the field (Figure 3 .

The magnetic flux $\Phi$ and the total flare energy $\mathrm{E}$ are defined as following:

$\phi=42\left(\frac{B_{z}}{8 T}\right)\left(\frac{L^{\text {bipole }}}{5 m}\right)^{2} \mathrm{~Wb}$

$\mathrm{E}=\frac{40}{\mu\left(\frac{B_{z}}{8 T}\right)^{2}\left(\frac{L^{b i p o l e}}{5 m}\right)^{3}} \mathrm{~J}$

$\mathrm{B}$ is the strength of the magnetic field in the bipole (sunspot), L is the size of the bipole. The problem is the estimation of the value L. $\mathrm{L}^{2}$ can be computed as the area of an active region with facula $(\mathrm{L}=200 \mathrm{Mm})$, The maximum value for the flux is $\phi=10^{23} \mathrm{Mx}$ and for the energy $\mathrm{E}=3 \times 10^{34} \mathrm{erg}$ that falls in the range of star superflares (Maehara et al. 2012). However L should be reduced to $1 / 3$ due to the fact that the stress of the field concerned only a small part of the PIL (Aulanier et al. 2013). The maximum of energy could not exceed $10^{34} \mathrm{erg}$. These results come from a self consistent model with shear flux 
leading to CME with no approximation. On the other hand the estimations of Toriumi et al. (2016) are very empirical mixing different observations not related one to the other one. Each estimation has been overestimated. For example the volume of the active region concerned by the flare has been estimated by the product of $\mathrm{S}_{\text {ribbon }}$ (surface area of the ribbons) and distance between the ribbons (Toriumi et al., 2016). However the uncertainty on the estimation of the magnetic field in this volume can lead to an overestimation by one to two orders of magnitude according to the $\mathrm{f}$ value introduced in their equations. Taking unrealistic values of B and flux lead to unrealistic energy values never observed in our era (Emslie et al. 2012).

\section{Conclusion}

Commonly extreme solar events are produced in active regions having a strong magnetic reservoir (high magnetic field and stress). There are defined as very powerful X ray flares, coronal mass ejections with high kinetic energy faced to the Earth leading to magnetic cloud arriving at the magnetosphere with a good orientation ( $\mathrm{B}_{z}$ negative) and strong ejections of energetic particles (SEPs). Large sunspot groups with fragmentation are good candidates for extreme solar storms (Sammis et al. 2000).

With our Sun as it is today, it seems impossible to get larger sunspots and super-flares with energy $>10^{34}$ erg. Figure 4 shows different sunspot groups. In each of them a pair of sunspot surrounded by red curves represents the bipole used as boundary condition of the OHM simulation. The energy mentioned below the pair is the result of the simulation. With huge sunspots we obtain large energies as it is recorded for stars by the Kepler satellite. Such large spots have never been observed on the Sun. We should not forget that the simulation concerns a bipole with rotating spots imposing a strong shear along the PIL. The shear is a necessary ingredient to have expulsions of CMEs in the simulation and also in the observations. In order to produce stronger flares the Sun-like stars should have a much stronger dynamo than the Sun and a rotation rate 
exceeding several days. The prediction of having extreme solar storms in 800 years would be very speculative.

Acknowledgements

The author would like to thank the organizers of the meeting Drs. Katya Georgieva and Kazuo Shiokawa to invite me in Varna for the VarSITI meeting in June 2016. I want to thank G.Aulanier for his fruitful comments on this work.

\section{References}

Aulanier, G., Démoulin, P., Grappin, R., 2005. Equilibrium and observational properties of line-tied twisted flux tubes. Astronomy and Astrophysics Journal 430, 1067-1087. doi:10.1051/0004-6361:20041519.

Aulanier, G., Démoulin, P., Schrijver, C.J., Janvier, M., Pariat, E., Schmieder, B., 2013. The standard flare model in three dimensions. II. Upper limit on solar flare energy. Astrophys. J. 549, A66. doi 10.1051/0004-6361/ 201220406, arXiv:1212.2086.

Aulanier, G., Torok, T., Demoulin, P., DeLuca, E.E., 2010. Formation of TorusUnstable Flux Ropes and Electric Currents in Erupting Sigmoids. Astrophys. J. 708, 314-333. doi $10.1088 / 0004-637 \mathrm{X} / 708 / 1 / 314$.

Baker, D.N., 2013. The Major Solar Eruptive Event in July 2012: Defining Extreme Space Weather Scenarios (Invited). AGU Fall Meeting Abstracts .

Bein, B.M., Berkebile-Stoiser, S., Veronig, A.M., Temmer, M., Muhr, N., Kienreich, I., Utz, D., Vršnak, B., 2011. Impulsive Acceleration of Coronal Mass Ejections. I. Statistics and Coronal Mass Ejection Source Region Characteristics. Astrophys. J. 738, 191. doi 10.1088/0004-637X/738/2/191, arXiv:1108.0561

Bosman, E., Bothmer, V., Nisticò, G., Vourlidas, A., Howard, R.A., Davies, J.A., 2012. Three-Dimensional Properties of Coronal Mass Ejections from 
STEREO/SECCHI Observations. Solar Phys. 281, 167-185. doi 10.1007/ s11207-012-0123-5.

Bothmer, V., Zhukov, A., 2007. The Sun as the prime source of space weather. Book. p. 31. doi:10.1007/978-3-540-34578-7_3.

Carrington, R.C., 1859. Description of a Singular Appearance seen in the Sun on September 1, 1859. Mon. Not. Roy. Astron. Soc. 20, 13-15. doi10.1093/ mnras/20.1.13.

Chandra, R., Pariat, E., Schmieder, B., Mandrini, C.H., Uddin, W., 2010. How Can a Negative Magnetic Helicity Active Region Generate a Positive Helicity Magnetic Cloud? Solar Phys. 261, 127-148. doi10.1007/ s11207-009-9470-2, arXiv:0910.0968.

Chiu, M.C., von-Mehlem, U.I., Willey, C.E., Betenbaugh, T.M., Maynard, J.J., Krein, J.A., Conde, R.F., Gray, W.T., Hunt, Jr., J.W., Mosher, L.E., McCullough, M.G., Panneton, P.E., Staiger, J.P., Rodberg, E.H., 1998. ACE Spacecraft. Space Sci. Rev. 86, 257-284. doi 10.1023/A:1005002013459.

Cid, C., Cremades, H., Aran, A., Mandrini, C., Sanahuja, B., Schmieder, B., Menvielle, M., Rodriguez, L., Saiz, E., Cerrato, Y., Dasso, S., Jacobs, C., Lathuillere, C., Zhukov, A., 2012. Can a halo CME from the limb be geoeffective? Journal of Geophysical Research (Space Physics) 117, 11102. doi:10.1029/2012JA017536.

Cid, C., Palacios, J., Saiz, E., Cerrato, Y., Aguado, J., Guerrero, A., 2013. Modeling the recovery phase of extreme geomagnetic storms. Journal of Geophysical Research (Space Physics) 118, 4352-4359. doi 10.1002/jgra.50409

Cliver, E.W., Dietrich, W.F., 2013. The 1859 space weather event revisited: limits of extreme activity. Journal of Space Weather and Space Climate 3, A31. doi $10.1051 / \mathrm{swsc} / 2013053$. 
Cliver, E.W., Svalgaard, L., 2004. The 1859 Solar-Terrestrial Disturbance And the Current Limits of Extreme Space Weather Activity. Solar Phys. 224, 407-422. doi:10.1007/s11207-005-4980-z.

Dere, K.P., Howard, R.A., Brueckner, G.E., 2000. Coronal Mass Ejections and the Solar Wind: New Results from LASCO. Advances in Space Research 25, 1837-1842. doi 10.1016/S0273-1177(99)00594-3.

Dodson, H.W., Hedeman, E.R., 1949. The Frequency and Positions of Flares Within Three Active Sunspot Areas. Astrophys. J. 110, 242. doi10.1086/ 145200 .

Echer, E., Gonzalez, W.D., Tsurutani, B.T., 2011a. Statistical studies of geomagnetic storms with peak dst le-50 nt from 1957 to 2008. Journal of Atmospheric and Solar-Terrestrial Physics 73, 1454-1459. doi $10.1016 / j \cdot j$ jastp. 2011.04.021.

Echer, E., Tsurutani, B.T., Gonzalez, W.D., 2013. Interplanetary origins of moderate (-100 nt dst -50 nt) geomagnetic storms during solar cycle 23 (19962008). Journal of Geophysical Research (Space Physics) 118, 385-392. doi:10. 1029/2012JA018086.

Echer, E., Tsurutani, B.T., Guarnieri, F.L., Kozyra, J.U., 2011b. Interplanetary fast forward shocks and their geomagnetic effects: CAWSES events. Journal of Atmospheric and Solar-Terrestrial Physics 73, 1330-1338. doi:10.1016/j. jastp.2010.09.020.

Emslie, A.G., Dennis, B.R., Shih, A.Y., Chamberlin, P.C., Mewaldt, R.A., Moore, C.S., Share, G.H., Vourlidas, A., Welsch, B.T., 2012. Global Energetics of Thirty-eight Large Solar Eruptive Events. Astrophys. J. 759, 71. doi:10.1088/0004-637X/759/1/71, arXiv:1209.2654.

Eren, S., Kilcik, A., Atay, T., Miteva, R., Yurchyshyn, V., Rozelot, J.P., Ozguc, A., 2017. Flare-production potential associated with different sunspot groups. Mon. Not. Roy. Astron. Soc. 465, 68-75. doi 10.1093/mnras/stw2742. 
Fleck, B., Domingo, V., Poland, A.I., 1995. The SOHO mission. Solar Phys. 162.

Gonzalez, W.D., Echer, E., Clúa de Gonzalez, A.L., Tsurutani, B.T., Lakhina, G.S., 2011a. Extreme geomagnetic storms, recent Gleissberg cycles and space era-superintense storms. Journal of Atmospheric and Solar-Terrestrial Physics 73, 1447-1453. doi $10.1016 / j \cdot j$ astp. 2010.07 .023

Gonzalez, W.D., Echer, E., Clua-Gonzalez, A.L., Tsurutani, B.T., 2007. Interplanetary origin of intense geomagnetic storms (Dst $=-100 \mathrm{nT})$ during solar cycle 23. Geophys. Res. Lett. 34, 6101. doi 10.1029/2006GL028879.

Gonzalez, W.D., Echer, E., Tsurutani, B.T., Clúa de Gonzalez, A.L., Dal Lago, A., 2011b. Interplanetary Origin of Intense, Superintense and Extreme Geomagnetic Storms. Space Sci. Rev. 158, 69-89. doi10.1007/ s11214-010-9715-2.

Gopalswamy, N., Yashiro, S., Liu, Y., Michalek, G., Vourlidas, A., Kaiser, M.L., Howard, R.A., 2005. Coronal mass ejections and other extreme characteristics of the 2003 October-November solar eruptions. Journal of Geophysical Research (Space Physics) 110, 9. doi:10.1029/2004JA010958.

Gopalswamy, N., Yashiro, S., Michalek, G., Stenborg, G., Vourlidas, A., Freeland, S., Howard, R., 2009. The SOHO/LASCO CME Catalog. Earth Moon and Planets 104, 295-313. doi:10.1007/s11038-008-9282-7.

Gopalswamy, N., Yashiro, S., Michalek, G., Xie, H., Mäkelä, P., Vourlidas, A., Howard, R.A., 2010a. A Catalog of Halo Coronal Mass Ejections from SOHO. Sun and Geosphere 5, 7-16.

Gopalswamy, N., Yashiro, S., Xie, H., Akiyama, S., Mäkelä, P., 2010 b. Large Geomagnetic Storms Associated with Limb Halo Coronal Mass Ejections. Advances in Geosciences, Volume 21: Solar Terrestrial (ST) 21, 71. arXiv:0903.2776 
Hodgson, R., 1859. On a curious Appearance seen in the Sun. Mon. Not. Roy. Astron. Soc. 20, 15-16. doi 10.1093/mnras/20.1.15.

Isavnin, A., Vourlidas, A., Kilpua, E.K.J., 2013. Three-Dimensional Evolution of Erupted Flux Ropes from the Sun $(2-20$ R ?) to 1 AU. Solar Phys. 284, 203-215. doi:10.1007/s11207-012-0214-3, arXiv:1211.2108.

Kilcik, A., Yurchyshyn, V.B., Abramenko, V., Goode, P.R., Ozguc, A., Rozelot, J.P., Cao, W., 2011. Time Distributions of Large and Small Sunspot Groups Over Four Solar Cycles. Astrophys. J. 731, 30. doi 10.1088/0004-637X/ 731/1/30, arXiv:1111.3999.

Kilpua, E.K.J., Pomoell, J., Vourlidas, A., Vainio, R., Luhmann, J., Li, Y., Schroeder, P., Galvin, A.B., Simunac, K., 2009. STEREO observations of interplanetary coronal mass ejections and prominence deflection during solar minimum period. Annales Geophysicae 27, 4491-4503. doi 10.5194/ angeo-27-4491-2009.

Koskinen, H.E.J., Huttunen, K.E.J., 2006. Geoeffectivity of Coronal Mass Ejections. Space Sci. Rev. 124, 169-181. doi 10.1007/s11214-006-9103-0.

Lakhina, G.S., Alex, S., Rawat, R., 2008. An Overview of the Magnetosphere, Substorms and Geomagnetic Storms. Astrophysics and Space Science Proceedings 12 , 293. doi $10.1007 / 978-1-4020-8868-1 \_20$

Lugaz, N., Temmer, M., Wang, Y., Farrugia, C.J., 2017. The Interaction of Successive Coronal Mass Ejections: A Review. Solar Phys. 292, 64. doi 10 . 1007/s11207-017-1091-6, arXiv:1612.02398.

Maehara, H., Shibayama, T., Notsu, S., Notsu, Y., Nagao, T., Kusaba, S., Honda, S., Nogami, D., Shibata, K., 2012. Superflares on solar-type stars. Nature 485, 478-481. doi $10.1038 /$ nature11063.

Marubashi, K., Cho, K.S., Kim, Y.H., Park, Y.D., Park, S.H., 2012. Geometry of the 20 November 2003 magnetic cloud. Journal of Geophysical Research (Space Physics) 117, 1101. doi:10.1029/2011JA016802. 
McIntosh, P.S., 1990. The classification of sunspot groups. Solar Phys. 125, 251-267. doi:10.1007/BF00158405

Mein, N., Schmieder, B., Simon, G., Tandberg-Hanssen, E., Wu, S.T., 1982. Dynamics of the eruptive prominence of 6 May 1980 and its relationship to the coronal transient. Astron. Astrophys. 114, 192-199.

Möstl, C., Miklenic, C., Farrugia, C.J., Temmer, M., Veronig, A., Galvin, A.B., Vršnak, B., Biernat, H.K., 2008. Two-spacecraft reconstruction of a magnetic cloud and comparison to its solar source. Annales Geophysicae 26, 3139-3152. doi:10.5194/angeo-26-3139-2008.

Möstl, C., Rollett, T., Frahm, R.A., Liu, Y.D., Long, D.M., Colaninno, R.C., Reiss, M.A., Temmer, M., Farrugia, C.J., Posner, A., Dumbović, M., Janvier, M., Démoulin, P., Boakes, P., Devos, A., Kraaikamp, E., Mays, M.L., Vršnak, B., 2015. Strong coronal channelling and interplanetary evolution of a solar storm up to Earth and Mars. Nature Communications 6, 7135. doi 10.1038/ ncomms8135, arXiv:1506.02842

Russell, C.T., 2008. The STEREO Mission. Space Sci. Rev. 136, 1-3. doi 10 . 1007/s11214-008-9344-1.

Sammis, I., Tang, F., Zirin, H., 2000. The Dependence of Large Flare Occurrence on the Magnetic Structure of Sunspots. Astrophys. J. 540, 583-587. doi 10 . $1086 / 309303$.

Shibata, K., Isobe, H., Hillier, A., Choudhuri, A.R., Maehara, H., Ishii, T.T., Shibayama, T., Notsu, S., Notsu, Y., Nagao, T., Honda, S., Nogami, D., 2013. Can Superflares Occur on Our Sun? Pub. Astron. Soc. Japan 65, 49. doi:10.1093/pasj/65.3.49, arXiv:1212.1361

Smith, E.J., 1976. The August 1972 solar-terrestrial events - Interplanetary magnetic field observations. Space Sci. Rev. 19, 661-686. doi 10.1007/ BF00210645. 
St. Cyr, O.C., Plunkett, S.P., Michels, D.J., Paswaters, S.E., Koomen, M.J., Simnett, G.M., Thompson, B.J., Gurman, J.B., Schwenn, R., Webb, D.F., Hildner, E., Lamy, P.L., 2000. Properties of coronal mass ejections: Soho lasco observations from january 1996 to june 1998. J. Geophys. Res. 105, 18169-18186. doi:10.1029/1999JA000381.

Sun, X., Bobra, M.G., Hoeksema, J.T., Liu, Y., Li, Y., Shen, C., Couvidat, S., Norton, A.A., Fisher, G.H., 2015. Why Is the Great Solar Active Region 12192 Flare-rich but CME-poor? Astrophys. J. Lett. 804, L28. doi $10.1088 /$ 2041-8205/804/2/L28, arXiv:1502.06950.

Thalmann, J.K., Su, Y., Temmer, M., Veronig, A.M., 2015. The Confined X-class Flares of Solar Active Region 2192. Astrophys. J. Lett. 801, L23. doi:10.1088/2041-8205/801/2/L23, arXiv:1502.05157.

Toriumi, S., Schrijver, C.J., Harra, L.K., Hudson, H., Nagashima, K., 2016. Magnetic Properties of Solar Active Regions that Govern Large Solar Flares and Eruptions. ArXiv e-prints arXiv:1611.05047.

Tsurutani, B.T., Gonzalez, W.D., Lakhina, G.S., Alex, S., 2003. The extreme magnetic storm of 1-2 September 1859. Journal of Geophysical Research (Space Physics) 108, 1268. doi:10.1029/2002JA009504.

Tsurutani, B.T., Gonzalez, W.D., Tang, F., Lee, Y.T., Okada, M., Park, D., 1992. Reply to L. J. Lanzerotti: Solar wind RAM pressure corrections and an estimation of the efficiency of viscous interaction. Geophys. Res. Lett. 19, 1993-1994. doi:10.1029/92GL02239.

Waldmeier, M., 1955. Provisional Sunspot-Numbers for July to September, 1955. J. Geophys. Res. 60, 525 .

Webb, D.F., Howard, T.A., 2012. Coronal mass ejections: Observations. Living Reviews in Solar Physics 9. URL: http://www.livingreviews.org/ lrsp-2012-3, doi:10.12942/lrsp-2012-3 
Wimmer-Schweingruber, R.F., 2014. Interplanetary Disturbances Affecting Space Weather, in: Schmieder, B., Malherbe, J.M., Wu, S.T. (Eds.), IAU Symposium, pp. 297-306. doi 10.1017/S1743921313011125.

Wuelser, J.P., Lemen, J.R., Tarbell, T.D., Wolfson, C.J., Cannon, J.C., Carpenter, B.A., Duncan, D.W., Gradwohl, G.S., Meyer, S.B., Moore, A.S., Navarro, R.L., Pearson, J.D., Rossi, G.R., Springer, L.A., Howard, R.A., Moses, J.D., Newmark, J.S., Delaboudiniere, J.P., Artzner, G.E., Auchere, F., Bougnet, M., Bouyries, P., Bridou, F., Clotaire, J.Y., Colas, G., Delmotte, F., Jerome, A., Lamare, M., Mercier, R., Mullot, M., Ravet, M.F., Song, X., Bothmer, V., Deutsch, W., 2004. EUVI: the STEREO-SECCHI extreme ultraviolet imager, in: Fineschi, S., Gummin, M.A. (Eds.), Telescopes and Instrumentation for Solar Astrophysics, pp. 111-122. doi:10.1117/12.506877.

Yashiro, S., Akiyama, S., Gopalswamy, N., Howard, R.A., 2006. Different PowerLaw Indices in the Frequency Distributions of Flares with and without Coronal Mass Ejections. Astrophys. J. Lett. 650, L143-L146. doi:10.1086/508876, arXiv:astro-ph/0609197.

Zhang, G., Burlaga, L.F., 1988. Magnetic clouds, geomagnetic disturbances, and cosmic ray decreases. J. Geophys. Res. 93, 2511-2518. doi10.1029/ JA093iA04p02511

Zhang, J., Richardson, I.G., Webb, D.F., Gopalswamy, N., Huttunen, E., Kasper, J.C., Nitta, N.V., Poomvises, W., Thompson, B.J., Wu, C.C., Yashiro, S., Zhukov, A.N., 2007. Solar and interplanetary sources of major geomagnetic storms (Dst $=-100 \mathrm{nT}$ ) during 1996-2005. Journal of Geophysical Research (Space Physics) 112, 10102. doi 10.1029/2007JA012321.

Zuccarello, F.P., Aulanier, G., Gilchrist, S.A., 2015. Critical Decay Index at the Onset of Solar Eruptions. Astrophys. J. 814, 126. doi 10.1088/0004-637X/ 814/2/126, arXiv:1510.03713.

Zuccarello, F.P., Bemporad, A., Jacobs, C., Mierla, M., Poedts, S., Zuccarello, F., 2012. The Role of Streamers in the Deflection of Coronal Mass Ejections: 
Comparison between STEREO Three-dimensional Reconstructions and Numerical Simulations. Astrophys. J. 744, 66. doi 10.1088/0004-637X/744/ $1 / 66$. 